\title{
Convex endobronchial ultrasound: same coin, two faces. Challenging biopsy and staging for non-small-cell lung cancer
}

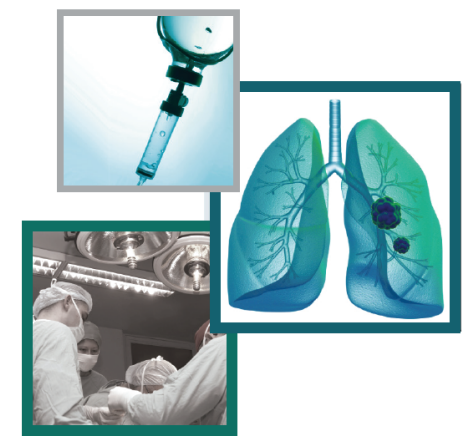

Konstantinos Sapalidis*,1, Konstantinos Romanidis², Panagoula Oikonomou², Paul Zarogoulidis 1,3, Athanasios Katsaounis', Aikaterini Amaniti ${ }^{4}$, Nikolaos Michalopoulos ${ }^{1}$, Charilaos Koulouris ${ }^{1}$, Kosmas Tsakiridis ${ }^{5}$, Dimitrios Giannakidis ${ }^{1}$, Isaak Kesisoglou ${ }^{1}$, Aris Ioannidis ${ }^{1}$, Iason Nikolaos-Katsios ${ }^{1}$, Anastasios Vagionas ${ }^{6}$, Wolfgang Hohenforst-Schmidt ${ }^{7}$, Haidong Huang ${ }^{8}$, Chong Bai ${ }^{1}$, Alexandru Marian Goganau ${ }^{9}$ \& Christoforos Kosmidis ${ }^{1}$

${ }^{1}$ 3rd Department of Surgery, AHEPA University Hospital, Aristotle University of Thessaloniki, Thessaloniki, Greece

2Second Department of Surgery, University Hospital of Alexandroupolis, Medical School, Democritus University of Thrace, Alexandroupolis, Greece

${ }^{3}$ Pulmonary Department, Creta InterClinic Private Hospital, Iraklio, Crete, Greece

${ }^{4}$ Anesthesiology Department, AHEPA University Hospital, Aristotle University of Thessaloniki, Thessaloniki, Greece

${ }^{5}$ Thoracic Surgery Department, Interbalkan European Medical Center, Thessaloniki, Greece

${ }^{6}$ Oncology Department, General Hospital of Kavala, Kavala, Greece

${ }^{7}$ Sana Clinic Group Franken, Department of Cardiology/Pulmonology/Intensive Care/Nephrology, 'Hof' Clinics, University of Erlangen, Hof, Germany

${ }^{8}$ The Diagnostic \& Therapeutic Center of Respiratory Diseases, Shanghai East Hospital, Tongji University, Shanghai, China

${ }^{9}$ General Surgery Clinic 1, University of Medicine andPharmacy of Craiova, Craiova County Emergency Hospital, Craiova, Romania

*Author for correspondence: sapalidiskonstantinos@gmail.com

\section{Practice points}

- Transbronchial needle aspiration with endobronchial ultrasounds for paraesophageal masses.

- General anesthesia with intubation makes the access of the esophagus very easy.

- General anesthesia with jet-ventilation makes the diagnostic process easier and safer for patients with chronic obstructive pulmonary disease.

- There is practically no major difference between the different ultrasound endoscopic equipment.

- Paraesophageal masses are easily accessible with transbronchial needle aspiration with endobronchial ultrasounds.

- It is not always necessary to use general anesthesia in order to obtain tissue sample.

Lung cancer is still diagnosed at a late stage due to lack of early disease symptoms. Despite the development of new diagnostic endoscopic tools, such as radial/convex endobronchial ultrasounds (EBUS) and electromagnetic navigation, most patients are still diagnosed at advanced stage disease. Most of the patients refer to their doctor only if they cough blood or their cough changes character. There are challenging cases in the diagnosis and staging of a patient, such as the one that we will present. We present a case of lung cancer that was diagnosed through a biopsy from the main lesion, with access from the esophagus, through transbronchial needle aspiration with EBUS, under general anesthesia and intubation. Staging with transbronchial needle aspiration with EBUS was also performed at the same session.

First draft submitted: 27 August 2019; Accepted for publication: 9 December 2019; Published online: 16 January 2020

Keywords: bronchoscopy $\bullet$ convex probe $\bullet$ EBUS $\bullet$ EUS $\bullet$ NSCLC

Nowadays, we have novel diagnostic tools for lung cancer with radial/convex endobronchial ultrasounds (EBUS) and electromagnetic navigation [1-3]. The radial probe is used for peripheral lesions while the convex probe is used for central and lymph node staging. Esophageal ultrasound (EUS), on the other hand, is used for the diagnosis of gastrointestinal lesions and lymph node staging of gastrointestinal disease. However, there are cases where the EUS endoscopic technique can be used to obtain a sample within the mediastinum and the convex-EBUS system can be used to obtain sample from paraesophageal lesions. The new convex-EBUS system has a larger working 


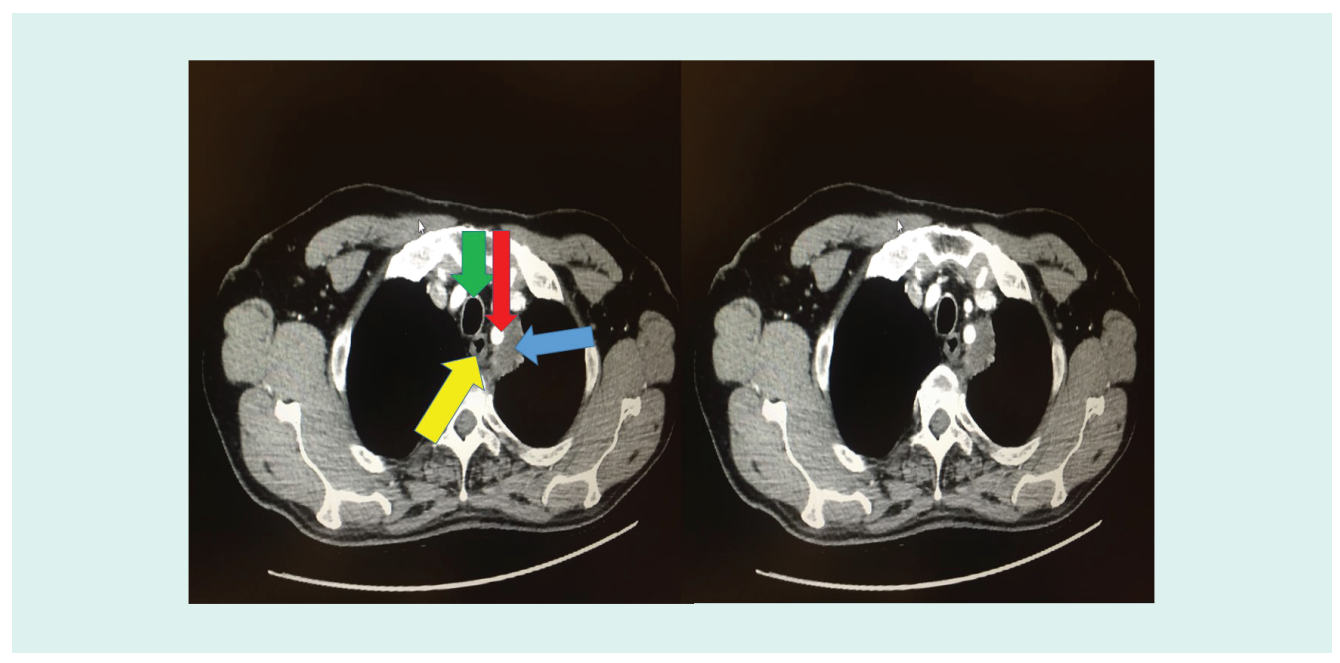

Figure 1. Computed tomography scan of the thorax with intravenous contrast administration on the day the endoscopy was performed. The green arrow indicates the trachea; the yellow arrow indicates the esophagus; the red arrow indicates the vessel; and the blue arrow indicates the mass.

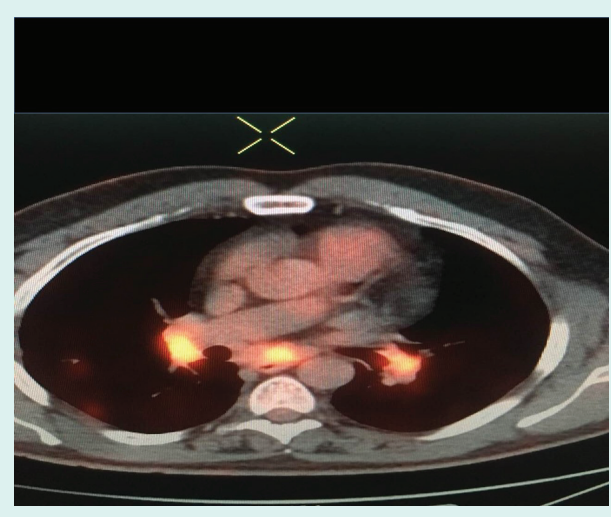

Figure 2. Positron emission computed tomography scan. Lymph node stations $10 \mathrm{R}, 7$ and $10 \mathrm{~L}$ are positive and therefore the $\mathrm{N}$ status of the disease was 3.

channel and, nowadays, the operator can use a larger, 19G needle without damaging the equipment (working channel). Until now, we used 21 and $22 \mathrm{G}$ needles and, by making patents, we used $19 \mathrm{G}$ out of the indications of the equipment.

On the other hand, due to the large working channel, the EUS system can use all three diameters: 19, 21 and 22G. We avoid using $19 \mathrm{G}$ needle for lesions $\leq 2 \mathrm{~cm}$ and for stations $4 \mathrm{~L}, 4 \mathrm{R}, 10 \mathrm{~L}$ and $10 \mathrm{R}$, due to the high probability of causing severe adverse effects [4]. In order to make better use of the material obtained from the 21 and $22 \mathrm{G}$ needle, we make cell blocks, but this is not necessary for the $19 \mathrm{G}$ needles, as they obtain larger tissue chunks [5]. Cell blocks can be used to investigate epidermal growth factor expression, anaplastic lymphoma kinase expression, programmed death-ligand, proto-oncogene B-Raf and proto-oncogene tyrosine-protein kinase [6-8]. Convex probe EBUS (CP-EBUS) is used both as a diagnostic tool for thoracic lesions and for non-small-cell lung cancer staging in conjunction with positron emission computed tomography (PET-CT) [9]. There are challenging cases, like the one that we present, where one instrument is used for both diagnosis and staging.

\section{Case presentation}

We present the case of a 50-year-old man referred to our interventional pulmonary department from our oncology department. The patient had a PET-CT scan and a CT scan of the thorax, which revealed a paraesophageal mass and that lymp nodes in the mediastinum were slightly positive $\geq 3 S U V$ (Figures $1 \& 2$ ). We intubated the patient and performed staging (stations $2 \mathrm{R} / \mathrm{L}, 4 \mathrm{R} / \mathrm{L}, 7,10 \mathrm{R} / \mathrm{L}$ and $11 \mathrm{R} / \mathrm{L}$ ) (Figure 3) and afterward, while the patient was still intubated, we inserted the CP-EBUS (PENTAX EB-1970UK, PENTAX Medical, Tokyo, Japan) through 


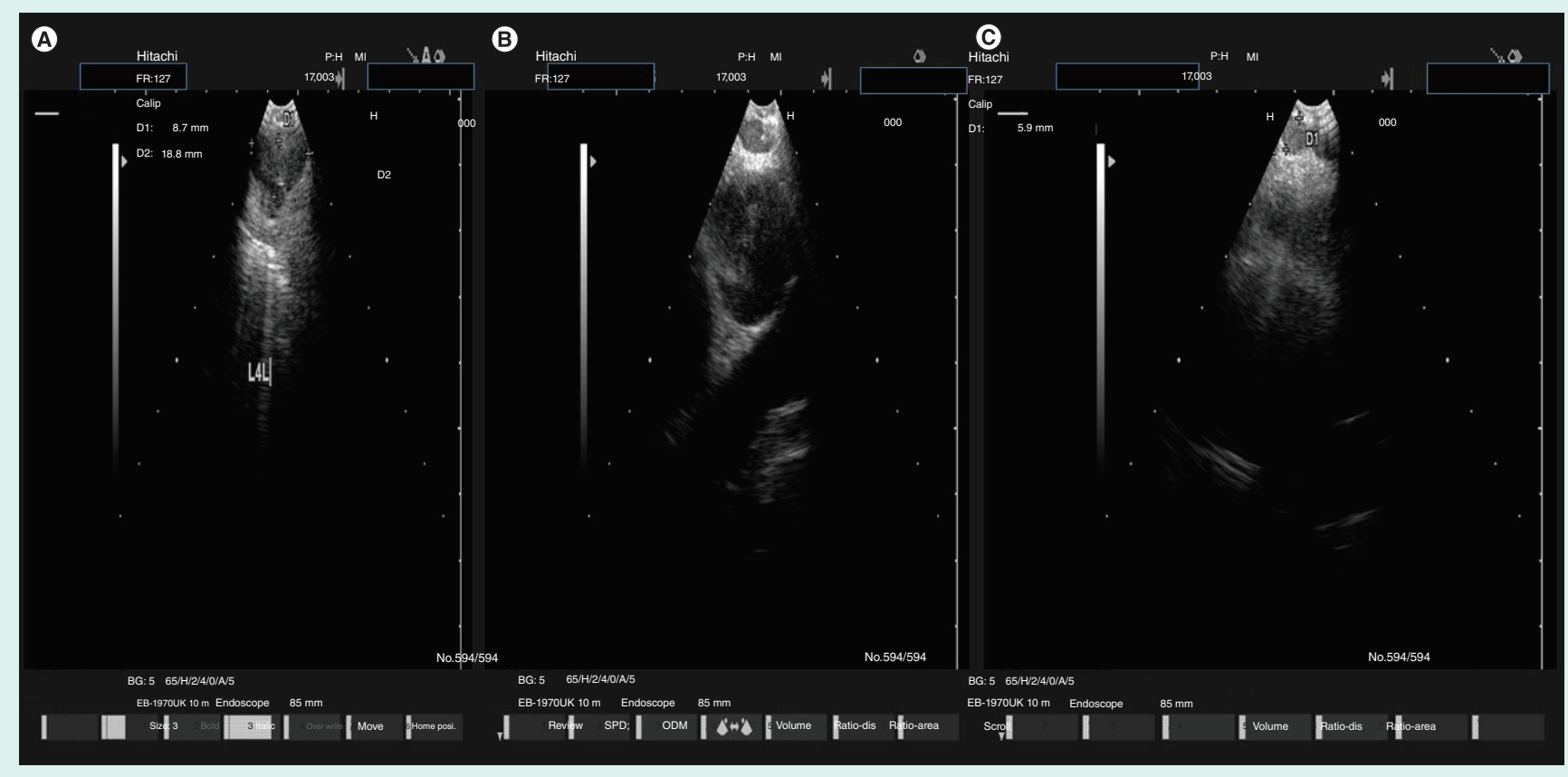

Figure 3. Ultrasound pictures taken during the procedure. We observe with the EUB $6500 \mathrm{HV}$ the ultrasound figures/lymph nodes, per station: (A) lymph node 4L, (B) lymph node 7 and (C) lymph node $11 \mathrm{~L}$.

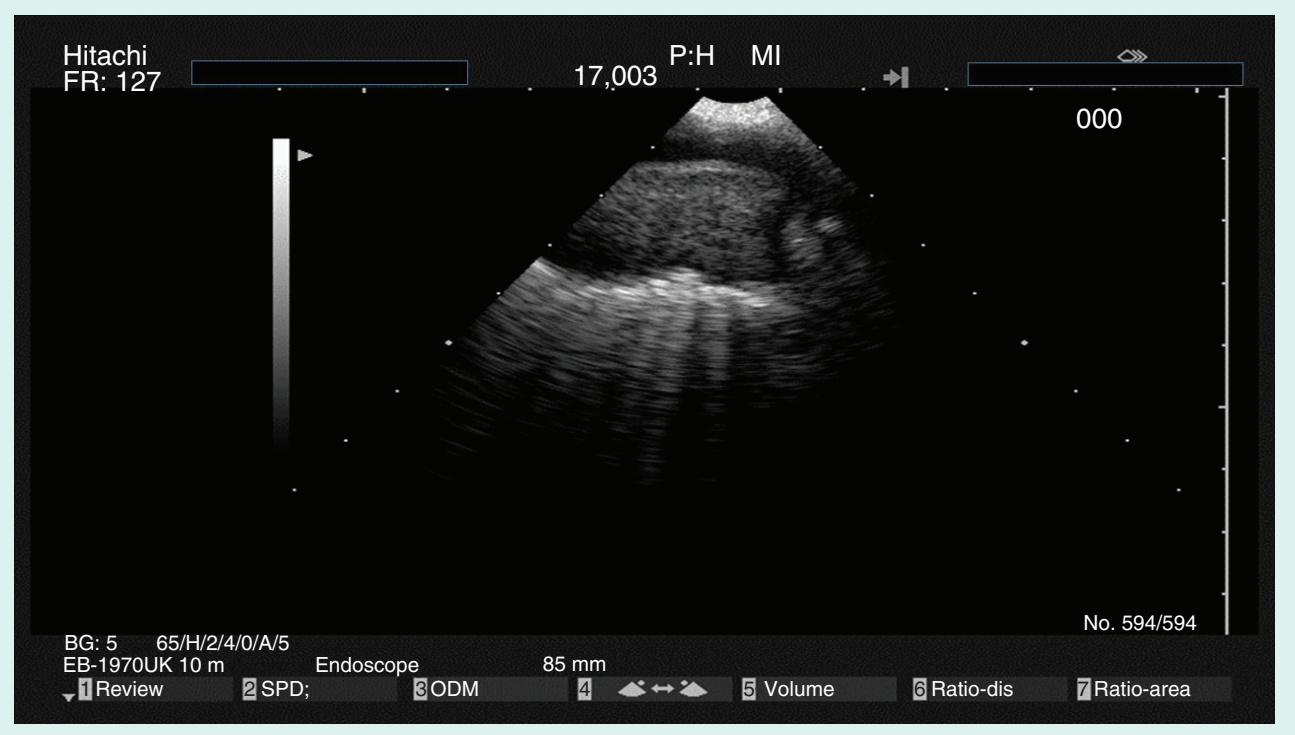

Figure 4. Paraesophageal mass imaged with the EUB-6500HV.

the esophagus and performed biopsy of the paraesophageal mass with a $22 \mathrm{G}$ needle (Figure 4). The patient did not have any intrabronchial lesions (Figure 5).

The patient was diagnosed with lung adenocarcinoma (Figure 6) with an unfortunate $\mathrm{N}$ category of N3, but he had $P D-L 1$ expression of $90 \%$ and received pembrolisumab as first-line treatment (Figure 7 ). We used the following staining protocol for PD-L1 IHC 22C3pharmDx DAKO. After deparaffinization and rehydration, tissue specimens were led in Target Retrieval Procedure with Target Retrieval Solution, Low pH (Code RT100/PT101/PT200, 

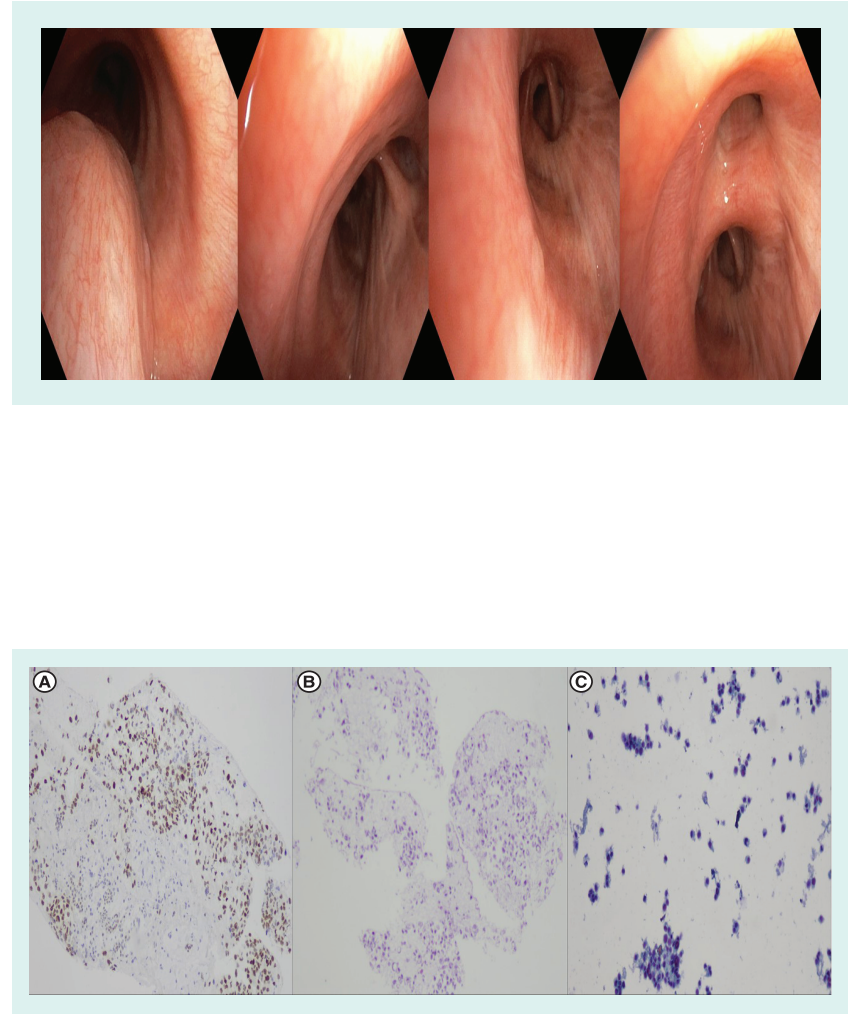

Figure 5. Representative bronchoscopic figures.

Figure 6. Tumor cells positive for (A) TTF1 x10, (B) cell block $\times 10$ and (C) pap smear $\times 10$. The cytological specimen was centrifuged and the material was placed in the cytoblock cassette. Additionally, the specimen was smeared onto glass slides. Cytologically, there were cuboidal or columnar malignant cells in low-cohesive aggregates. Single cells were also present. The nuclei were hyperchromatic and enlarged, with increased nuclear/cytoplasmic ratios. The nuclear membranes were well-defined and irregular. Immunohistochemically, the tumor cells were positive for TTF-1 and negative for $\mathrm{p} 40$. Based on cytomorphological and immunohistochemical findings, the final diagnosis was that of a lung adenocarcinoma.

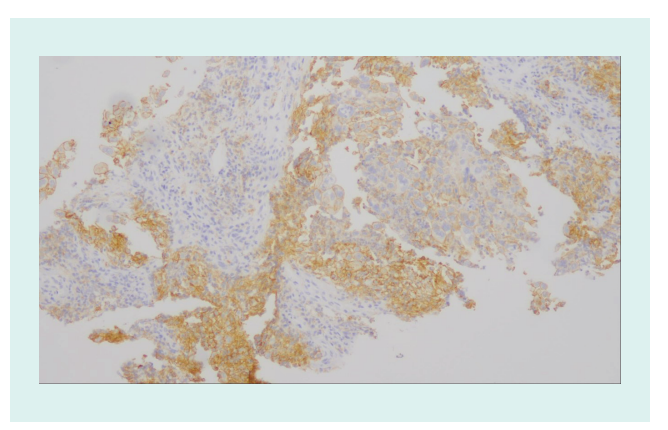

Figure 7. $P D-L 1$ positive case with whole membranous staining (intense $1+$ and $2+$ ) in $90 \%$ of neoplastic cells.

Dako, Agilent, CA, USA) for $20 \mathrm{~min}$ at $66^{\circ} \mathrm{C}$. The Autostainers racks with the specimen's slides were placed on the Autostainer Link 48 (Dako). The instrument performed the staining process by applying the appropriate reagent, monitoring the incubation time and rinsing slides between reagents. The reagents times were preprogrammed in the Dako Link software. Counterstain was performed with Hematoxyline (Link) (CodeK8008) and this was mounted with nonaqueous, permanent media.

\section{Discussion}

The CP-EBUS system can be used for the diagnosis of paraesophageal lesions and has been used on several occasions [10,11]. Based on the expertise of every pulmonary center, this approach is feasible and safe [12]. We used a $22 \mathrm{G}$ needle, which is known for its small diameter but is very effective for the diagnosis of several malignancies or benign lesions. Moreover, the material obtained, which is converted to cell block, can be used for the investigation of gene expression and certainly PD-L1 expression, as in our case [13-15]. The need for proper staging is absolutely necessary and therefore a combination of PET-CT and CP-EBUS is needed in every center for lung cancer health excellence. Currently, there are centers of pulmonary medicine where the CP-EBUS is used to obtain samples from the left adrenal gland, which is again feasible is many patients, with the exception of very tall patients [16]. The EUS, on the other hand, can be used as a diagnostic tool for the mediastinum whenever a lesion can be approached more efficiently and safely [17]. In our case, it was necessary to use the CP-EBUS through the esophagus for biopsy 
from the main lesion, since we could not approach it through the trachea. Moreover, we are currently investigating whether the use of EUS is necessary for the staging of mesothelioma [18]. In any case, several instruments can be used for diagnosis despite that not being their original intended purpose, as long as safety is established [19]. Advanced techniques should only be performed in centers of health excellence.

\section{Acknowledgments}

The authors would like to thank D Chatzibougias and lota Fitili of 'Microdiagnostics' Private Pathology Lab, Thessaloniki, Greece.

\section{Financial \& competing interests disclosure}

The authors have no relevant affiliations or financial involvement with any organization or entity with a financial interest in or financial conflict with the subject matter or materials discussed in the manuscript. This includes employment, consultancies, honoraria, stock ownership or options, expert testimony, grants or patents received or pending, or royalties.

No writing assistance was utilized in the production of this manuscript.

\section{Ethical conduct of research}

Patient consent was obtained in order to publish all relevant data of the case report.

\section{Open access}

This work is licensed under the Attribution-NonCommercial-NoDerivatives 4.0 Unported License. To view a copy of this license, visit http://creativecommons.org/licenses/by-nc-nd/4.0/

\section{References}

Papers of special interest have been highlighted as: $\bullet$ of interest; $\bullet \bullet$ of considerable interest

1. Zaric B, Stojsic V, Carapic V et al. Radial endobronchial ultrasound (EBUS) guided suction catheter-biopsy in histological diagnosis of peripheral pulmonary lesions. J. Cancer 7(1), 7-13 (2016).

2. Zarogoulidis $\mathrm{P}$, Huang $\mathrm{H}$, Bai $\mathrm{C}$ et al. A new mode of ventilation for interventional pulmonology. A case with EBUS-TBNA and debulking. Respir. Med. Case Rep. 23, 38-42 (2019).

3. Zaric B, Stojsic V, Sarcev T et al. Advanced bronchoscopic techniques in diagnosis and staging of lung cancer. J. Thorac. Dis. 5(Suppl. 4), S359-S370 (2013).

- Includes all diagnostic techniques for lung cancer.

4. Dooms C, Vander Borght S, Yserbyt J et al. A randomized clinical trial of flex 19G needles versus 22G needles for endobronchial ultrasonography in suspected lung cancer. Respiration 96(3), 275-282 (2018).

-. Indicates which biopsy needles can be used for each patient.

5. Pickering EM, Holden VK, Heath JE, Verceles AC, Kalchiem-Dekel O, Sachdeva A. Tissue acquisition during EBUS-TBNA: comparison of cell blocks obtained from a 19G versus $21 \mathrm{G}$ needle. J. Brochology Interv. Pulmonol. 26(4), 237-244 (2018).

-. Indicates why cell blocks are so important for needle samples.

6. Turner SR, Buonocore D, Desmeules P et al. Feasibility of endobronchial ultrasound transbronchial needle aspiration for massively parallel next-generation sequencing in thoracic cancer patients. Lung Cancer 119, 85-90 (2018).

-. The sample from needles can be used in order to perform gene investigation.

7. Demirci NY, Dikmen AU, Abdullayeva Z, Ozturk C. Contribution of cell blocks obtained through endobronchial ultrasound-guided transbronchial needle aspiration for the determination of lung cancer subtypes. Clin. Respir. J. 12(4), 1623-1627 (2018).

8. Hopkins E, Moffat D, Parkinson I et al. Cell block samples from endobronchial ultrasound transbronchial needle aspiration provide sufficient material for ancillary testing in lung cancer-a quaternary referral centre experience. J. Thorac. Dis. 8(9), 2544-2550 (2016).

9. Ozturk A, Gullu YT. Excellence in non-small-cell lung cancer staging by endobronchial-TBNA: comparison with PET-CT and surgery. Minim. Invasive Ther. Allied Techol. 28(4), 213-219 (2018).

10. Zarogoulidis P, Laskou S, Katsaounis A et al. Esophagus lyomyoma diagnosed with convex endobronchial ultrasound (EBUS). Respir. Med. Case Rep. 24, 95-97 (2018).

11. Skovgaard Christiansen I, Kuijvenhoven JC, Bodtger U et al. Endoscopic ultrasound with bronchoscope-guided fine needle aspiration for the diagnosis of paraesophageally located lung lesions. Respiration 97(4), 277-283 (2019).

- Previously published case with almost the same technique.

12. Wimaleswaran H, Farmer MW, Irving LB, Jennings BR, Steinfort DP. Pulmonologist-performed transoesophageal sampling for lung cancer staging using an endobronchial ultrasound video-bronchoscope: an Australian experience. Intern. Med. J. 47(2), 205-210 (2017).

- Previously published case with a very similar technique. 
13. Franco Serrano J, Bures Sales E. The contribution of cell blocks in the diagnosis of mediastinal masses and hilar adenopathy samples from echobronchoscopy. Arcch. Bronconeumol. 52(2), 107 (2016).

14. Raniszewska A, Polubiec-Kownacka M, Rutkowska E, Domagala-Kulawik J. PD-L1 expression on lung cancer stem cells in metastatic lymph nodes aspirates. Stem Cell Rev. Rep. 15(2), 324-330 (2019).

15. Labarca G, Folch E, Jantz M, Mehta HJ, Majid A, Fernandez-Bussy S. Adequacy of samples obtained by endobronchial ultrasound with transbronchial needle aspiration for molecular analysis in patients with non-small-cell lung cancer. Systematic review and meta-analysis. Ann. Am. Thorac. Soc. 15(10), 1205-1216 (2018).

16. Crombag LM, Annema JT. Left adrenal gland analysis in lung cancer patients using the endobronchial ultrasound scope: a feasibility trial. Respiration 91(3), 235-240 (2016).

17. Bendzsak A, Oliveira R, Goudie E et al. Evaluation of the mediastinum: differentiating between stations 4L, 5 , and 6 using EBUS and EUS. Ann. Thorac. Surg. 103(2), e219-e221 (2017).

- Describes which stations can be accessed with esophageal ultrasound and transbronchial needle aspiration with endobronchial ultrasound.

18. Rice DC, Steliga MA, Stewart J et al. Endoscopic ultrasound-guided fine needle aspiration for staging of malignant pleural mesothelioma. Ann. Thorac. Surg. 88(3), 862-868 (2009).

19. Fuso L, Varone F, Magnini D, Calvello M, Lo Greco E, Richeldi L. Ultrasonography of the mediastinum: techniques, current practice, and future directions. Respir. Care 63(11), 1421-1438 (2018).

-• Very important manuscript for understanding ultrasonography. 